\title{
Rose Essential Oil Extraction from Fresh Petals Using Synergetic Microwave \& Ultrasound Energy: Chemical Composition and Antioxidant Activity Assessment
}

\author{
Mariana Patrascu ${ }^{1,2}$ and Marilena Radoiu ${ }^{2}$ \\ 1. R\&D Department, Sintchem Technologies Ltd, Codlea, Brasov, Romania \\ 2. R\&D Department, ChemspeedLtd., Bucharest, Romania
}

\begin{abstract}
The aim of the present work was to investigate the synergetic effect of microwave and ultrasound treatment on the production, chemical composition and antioxidant activity of rose essential oil. The rose essential oil was extracted from fresh Rosax damascena Mill. petals by four methods, hydrodistillation, steam distillation, organic solvent extraction and ultrasounds followed by microwave hydrodistillation. The chemical composition of the extracts was analysed by GC-MS, and the antioxidant capacity by DPPH. It was found that both chemical composition and the antioxidant activity of the extracts depend on the extraction method. Overall it was found that microwaves coupled with ultrasonic treatment can be used effectively for the intensification of the extraction of monoterpenes and sesquiterpenes - fragrance bearing molecules - and equally, for increased antioxidant activity while using about 4 time shorter extraction time. The scale-up of the method was also evaluated. The results obtained in this research support the possible use of the US/MW method for the extraction of rose essential oil for the pharmaceutical and fragrance industry.
\end{abstract}

Key words: $2.45 \mathrm{GHz}$ microwaves, ultrasounds, rose petals, essential oil, scale-up, hydrodistillation.

\section{Introduction}

Extraction of natural antioxidant and antimicrobial active chemical compounds from aromatic and medicinal plants is one of the most important research areas for pharmaceutical and chemical industries. Among the natural compounds, essential oils are widely used as food flavours, constituents of fragrances and raw materials for the pharmaceutical industry [1]. Volatile oils have been shown to possess antibacterial, antifungal, antiviral, insecticidal and antioxidant properties $[2,3]$.

Essential oils are commonly found in dietary and other plants, and they have been reported to exhibit numerous biological effects: antioxidant [4, 5], anti-inflammatory and anti-carcinogenic [6]. These compounds constitute a very diverse group of secondary plant metabolites and they are further

Corresponding author: Marilena Radoiu, Ph.D., research fields: chemical synthesis, food processing. divided into subclasses according to their chemical structure $[7,8]$.

Various extraction methods are used in the manufacture and extraction of essential oils; the method used is normally dependant on what type of botanical material is being used. With other words, it is the type of plant material that determines which method will be used to obtain the essential oil. But there are exceptions, for instance, supercritical $\mathrm{CO}_{2}$ is a great way to extract most oils, but the cost involved in following this method is very high. Traditional techniques-distillation, solvent extraction, cold pressing - are associated with longer extraction times and lower yields, use of large amount of organic solvents and poor extraction efficiency. Although the extraction of essential oils may sound only to be of technical interest, it is one of the key points which determines the quality of the oil since a wrong, or wrongly executed extraction, can damage the oil and alter its chemical signature. 
Rose essential oil is an exceptionally rich source of different terpenic and sesquiterpenic compounds, which are mostly responsible for rose oil high bioactivity exploited in traditional and modern medicine [9]. Antioxidant activity of rose essential oil justifies its use in a broad range of applications, including cosmetics, nutraceuticals, phyto-medicine and pharmaceutical industry $[10,11]$.

In recent years, new extraction techniques of essential oil and bioactive compounds such as microwave-assisted extraction (MW) and ultrasound-assisted extraction (US) became very popular, because in comparison with conventional methods involving solvent extraction, these new techniques considerably increase the speed and efficiency of the process, being considerate to the principle of the green chemistry and selective extraction of thermo fragile and bioactive compounds from plants [12].

The field of microwave-assisted extraction of natural compounds is quite young. In the last two decades, new investigations have been prompted by an increasing demand of more efficient extraction techniques, amenable to automation: shorter extraction times, reduced organic solvent consumption, energy and costs saved, were the main tasks pursued. Driven by these goals, advances in microwave extraction have resulted in a number of innovative techniques such as microwave assisted solvent extraction, vacuum microwave hydro-distillation, microwave Soxhlet extraction, microwave-assisted Clevenger distillation, compressed air microwave distillation, microwave headspace extraction, microwave hydro-diffusion and gravity, and solvent-free microwave extraction. One of the success stories of the twenty first Century has been the partial replacement of conventional extraction processes, with green procedures (reducing energy, time, solvent, and waste) based on microwave irradiation.

While US can be effectively used to improve the extraction rate by increasing the mass transfer rates and possible rupture of cell wall due to formation of microcavities, the fundamentals of MW extraction process are based on the changes in the cell structure caused by electromagnetic waves.

Disadvantages of MW extraction are directly related to nonhomogeneous heating $[13,14]$ and may be overcome by the use of MW and US together $[15,16]$. However, in the many published papers comparing MW extraction with other advanced and conventional extraction methods, MW extraction has been accepted as a potential and powerful alternative for the extraction of organic compounds from plant materials.

\section{Experimental}

\subsection{Materials and Analysis}

Rosa x damascena Mill. petals were collected in May-July 2015 from Transylvanian ecological crop. Petals were separated from the plant and weighed in $200 \mathrm{~g}$ working batches and mixed with $1.8 \mathrm{~kg}$ of distilled water. All extractions were carried out in a 3 L Pyrex round bottom flask heated either by an electric jacket (hydrodistillation and hexane extraction) or by US/MW. The chemical composition of the extracted essential oil was determined via GC-MS chromatography on a Perkin Elmer Clarus 500 in conformity with standard ISO 9842: 2003-Oil of rose (Rosa $x$ damascena Miller) and the antioxidant activity by DPPH antioxidant assay method [17,18]. DPPH (2,2-diphenyl-1-picryl-hydrazyl-hydrate) free radical method is an antioxidant assay based on electron-transfer; when DPPH-free radical stable at room temperature reacts with an antioxidant compound, which can donate hydrogen, it is reduced. The changes in colour of DDPPH in ethanol (from deep purple to light yellow) were read at $517 \mathrm{~nm}$ after 30 min of reaction using a UV-VIS spectrophotometer. $50 \mu \mathrm{L}$ of five concentrations $(5,10,15,20$ and 25 $\mu \mathrm{g} / \mathrm{mL}$ ) of the extracted oil were added to $5 \mathrm{~mL}$ of a $0.005 \%$ (w/v) methanol solution of DPPH. The titration value in $\mathrm{mg} / \mathrm{g}$ was used to evaluate the antioxidant activity of the extracted oils. 


\subsection{US/MW Experimental Setup}

The US pretreatment of the extraction mixture was performed using a titanium Banry Sonic Ltd. Ultrasonic probe $20 \mathrm{kHz}$, max. $500 \mathrm{~W}$. The ultrasonic probe was inserted directly in to the Pyrex flask containing the extraction mixture $(1.8 \mathrm{~kg}$ water $+200 \mathrm{~g}$ fresh rose petals) and run for $15 \mathrm{~min}$ at $500 \mathrm{~W}, 40^{\circ} \mathrm{C}$. To avoid the increase of temperature above the set $40{ }^{\circ} \mathrm{C}$, a water cooled thermostat jacket (water at $4{ }^{\circ} \mathrm{C}$ ) was used. The Pyrex flask was immediately transferred to the equipment used for the microwave extraction.MW extraction of rose essential oil was performed and optimized in Sairem's Minilabotron 2000 consisting of a $2.45 \mathrm{GHz}$ microwave multimode resonant cavity equipped with a built-in $\mathrm{CW}$ (continuous wave) industrial generator (switch mode power supply and magnetron) with output power adjustable up to 2,000 W [19] (Fig. 1). The temperature was measured and controlled via fibre optic while the reaction mixture was stirred with an overhead mechanical agitator (stirrer shaft made out of glass). During operation, the Minilabotron allows the measurement and displays the values of forward and reflected power making easy to calculate the total power (energy) absorbed by the

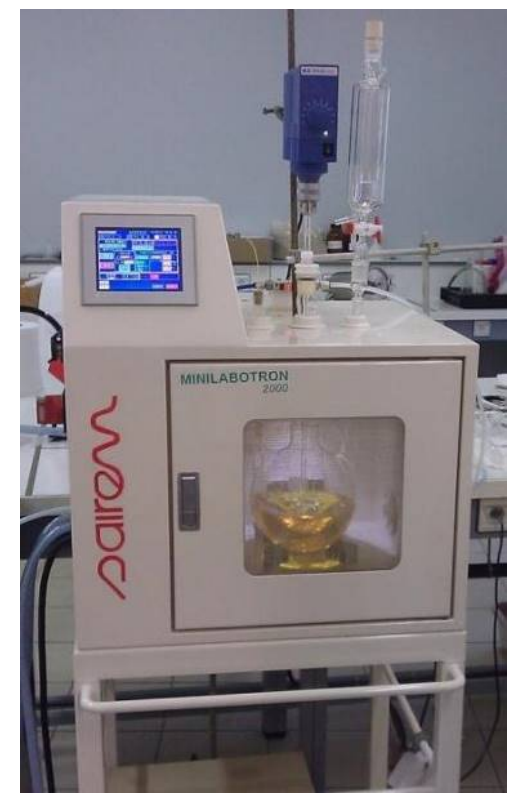

Fig. 1 Microwave equipment-Minilabotron 2000 with mechanical stirrer. reaction mixture and hence, to obtain reproducible extraction conditions. The Minilabotron was operated in temperature control mode, meaning that the microwave forward power was set and remained fixed at $1,000 \mathrm{~W}$ until the extraction medium $(1.8 \mathrm{~kg}$ water $+0.2 \mathrm{~kg}$ fresh rose petals) reached the boiling temperature, approx. $12 \mathrm{~min}$; afterwards, the microwave generator automatically reduced or cut off the forward power as such as enough energy was transferred to the extraction mixture to maintain a constant distillation rate until the end. The reflected power during the heating \& extraction steps was very low and therefore neglected.

\section{Results and Discussion}

The fundamentals of the microwave extraction process are different from those of conventional methods (solid-liquid or simply extraction) because the extraction occurs as the result of changes in the cell structure caused by electromagnetic waves [20]. The volumetric heating or heating of the bulk as opposed to transferring heat from the surface, inwards, is more efficient, uniform and less prone to overkill. Controllability is by far the greatest advantage of microwaves over conventional thermal technologies. In processing applications, the ability to instantaneously shut the heat source makes enormous difference to the product quality and expectedly, to the production economics.

The essential oils from rose petals were extracted via four methods, distillation with water (hydrodistillation) and steam, hexane extraction and US/MW extraction.

In each experiment, $200 \mathrm{~g}$ fresh rose petals and 1.8 $\mathrm{kg}$ distilled water or hexane were added into a round bottom flask and extracted via one of the four techniques listed in Table 1. The extracted oil was collected, dried over anhydrous sodium sulphate and stored at $4{ }^{\circ} \mathrm{C}$ until analysed. In the case of hexane extraction, the extracted oil was separated from hexane via rotary evaporation. 
Table 1 Extraction conditions of rose essential oil from Rosa damascena Mill; atmospheric pressure, rose petals $200 \mathrm{~g}$ (water or hexane: rose petals $=10: 1 \mathrm{w} / \mathrm{w}$ ).

\begin{tabular}{llll}
\hline Extraction method & Distillation system & $\begin{array}{l}\text { Distillation temperature } \\
\left({ }^{\circ} \mathrm{C}\right)\end{array}$ & $\begin{array}{l}\text { Distillation time } \\
(\mathrm{min})\end{array}$ \\
\hline Hydrodistillation & Clevenger & 100 & 240 \\
Steam distillation & Clevenger & 105 & 240 \\
Hexane extraction & Reflux & 70 & 240 \\
US/MW hydrodistillation & Clevenger & max. 98 & 60 \\
\hline
\end{tabular}

Table 2 Physical properties of rose essential oil vs. extraction method.

\begin{tabular}{llllll}
\hline Extraction method & Colour & $\begin{array}{l}\text { Density } \\
\left(\mathrm{g} / \mathrm{cm}^{3}\right)\end{array}$ & $\begin{array}{l}\text { Refraction } \\
\text { index }\end{array}$ & $\begin{array}{l}\text { Rotation index } \\
\left({ }^{\circ}\right)\end{array}$ & Standard characterisation method \\
\cline { 2 - 5 } & Yellow & 0.852 & 1.458 & -2.8 & $\begin{array}{l}\text { ISO 279:1998, Essential } \\
\text { Hydrodistillation }\end{array}$ \\
$\begin{array}{l}\text { Steam distillation } \\
\text { Hexane extraction }\end{array}$ & Transparent & 0.857 & 1.462 & -3.1 & $\begin{array}{l}{ }^{\circ} \mathrm{C} \\
\text { Yellow }\end{array}$ \\
$\begin{array}{llll}\text { US/MW } \\
\text { hydrodistillation }\end{array}$ & 0.856 & 1.456 & -3.4 & $\begin{array}{l}\text { ISO 280:1998, Essential oils } \\
\text { Pale yellowination of refractive index }\end{array}$ \\
\hline
\end{tabular}

Table 2 presents the standard characterisation method and the values obtained for four physical parameters of the extracted oil. As seen from Table 2, the use of US/MW extraction method caused no considerable change in the main physical properties.

The GC/MS analysis of rose oil extracted via steam (SD) \& water distillation (HD), hexane (HE) and US/MW revealed the presence of eleven main compounds divided in three main classes-oxygenated terpenes, sesqiterpenes and alkanes-representing over $80 \%$ of the total composition, Table 3. It can be seen that the US/MW method gives a higher composition of the desirable mono- and sesqiterpenes, which contribute mainly to the perfumery value of rose oil, i.e. $\sim 5-10 \%$ higher than the other three conventional methods, while the hydrocarbon (alkanes) content is lower. The ratio citronellol to geraniol, used for the evaluation of odour quality of the rose oil, is very similar for all extractions involving water as solvent (HD, SD and US/MW) and lower for the organic solvent extraction, i.e. 1.22 for $\mathrm{HD}, 1.22$ for SD, 1.21 for US/MW and 1.09 for HE. Citronellol and nonadecane were the main components in the essential oil extracted from rose petals but their corresponding relative amounts differed for the four extraction methods. Nonadecane is the most abundant hydrocarbon present in the rose oil. Higher amounts of nonadecane are present in the essential oil of rose petals extracted by HD and SD in comparison with SE and US/MW (HD 18.5\%, SD $15.5 \%$, HE $13.5 \%$, US/MW 11.9\%) although the total content of alkanes is very similar for the four tested extraction methods.

The antioxidant activity of the essential oils obtained via the above described extraction methods was evaluated from and correlated to the DPPH titration values (Table 4). As expected, the highest antioxidant activity was measured for the US/MW extract knowing that the higher the concentration of oxygenated monoterpenes (alcohols) and/or sesquiterpenes the higher the antioxidant properties. Similarly, the yield of the extracted oil was highest when using US/MW.

\section{Scale-up of US/MW Extraction}

The results obtained at laboratory scale and the availability of a pilot microwave equipment encouraged us to test the microwave extraction at a larger scale. Sairem's Labotron X6000 equipped with INTLI technology (internal transmission line) together with the U-waveguide [21] was used (Fig. 2). The principle of the MW processing using INTLI is to bring very 
Table 3 GC-MS chemical composition (\%) of rose essential oil depending on the extraction method.

\begin{tabular}{lllll}
\hline Chemical compound & Hydrodistillation & Steam distillation & Hexane extraction & US/MW hydrodistillation \\
\hline Citronellol & 23.36 & 23.04 & 20.16 & 24.16 \\
Nerol & 6.30 & 7.59 & 8.53 & 8.64 \\
Geraniol & 19.07 & 18.87 & 18.62 & 20.04 \\
Oxygenated terpenes & 48.73 & 49.50 & 47.31 & 52.84 \\
Caryophyllene & 3.07 & 3.24 & 4.14 & 2.62 \\
Farnesol & 2.55 & 2.72 & 3.92 & 2.14 \\
Sesquiterpenes & 5.62 & 5.96 & 8.06 & 4.76 \\
Nonadecane & 14.78 & 12.42 & 10.78 & 9.59 \\
Eicosane & 2.87 & 3.4 & 3.21 & 1.84 \\
Heneicosane & 3.45 & 2.23 & 3.54 & 4.54 \\
Tricosane & 2.67 & 4.12 & 3.43 & 3.82 \\
Heptacosane & 1.67 & 2.42 & 3.32 & 2.63 \\
Alkanes & 25.44 & 24.59 & 24.28 & 22.42 \\
\hline
\end{tabular}

Table 4 Effect of extraction technique on the antioxidant activity and extracted oil yield.

\begin{tabular}{lll}
\hline Extraction method & DPPH $(\mathrm{mg} / \mathrm{g})$ & Rose oil yield (\%) \\
Hydrodistillation & 2.1 & 0.05 \\
Steam distillation & 2.2 & 0.09 \\
Hexane extraction & 1.8 & 0.06 \\
US/MW hydrodistillation & 3.1 & 0.12 \\
\hline
\end{tabular}

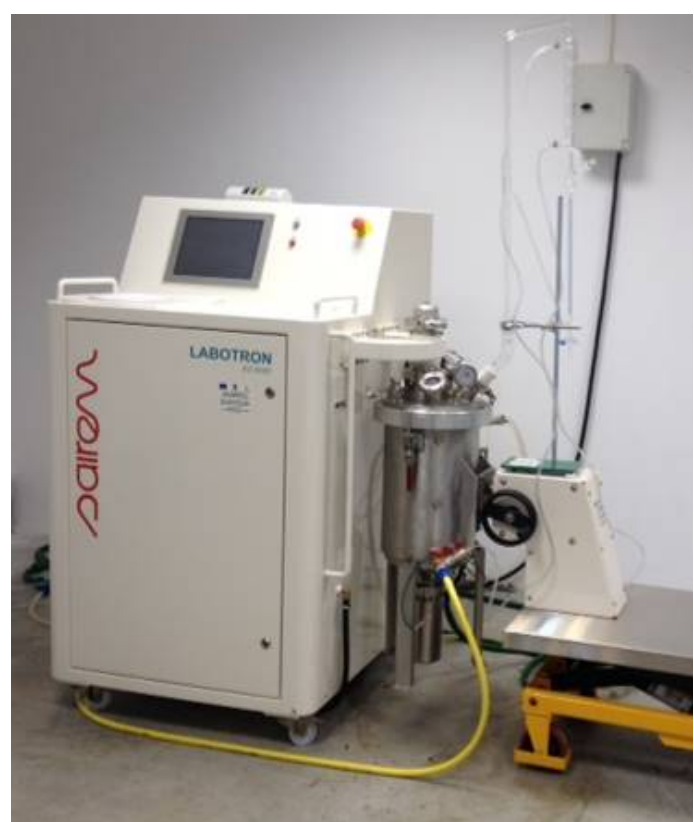

Fig. 2 The Labotron $\mathrm{X6000}$ and the stainless steel extractor with Clevenger.

selectively a great quantity of energy directly into the reaction mixture using high-density electromagnetic fields automatically adapted to each reaction mixture and, as such, making available very high densities of energy/heating which may be beneficial for plant extraction in solvents. The Labotron X6000 microwave extractor operates at $2.45 \mathrm{GHz} \mathrm{CW}$, max. MW output power $6,000 \mathrm{~W}$ (adjustable from $600 \mathrm{~W}$ to $6000 \mathrm{~W}$ ); the equipment has built-in impedance autotuning, fibre optic for temperature measurement and control, mechanical stirrer with adjustable speed; the stainless steel extractor used in these tests has a built-in cooling jacket which allows to both maintain the $40^{\circ} \mathrm{C}$ level during the initial sonication period (when necessary) and also to cool down rapidly the extraction mixture at the end of the treatment time.

The new INTLI method of introducing the microwave energy into the extractor in direct contact with the extraction mixture allowed to test a slightly more concentrated rose petals + water mix. Due to the low power of the ultrasound probe $(500 \mathrm{~W})$, an initial mix consisting of $3 \mathrm{~kg}$ rose petals and $5 \mathrm{~L}$ of water was sonicated for $20 \mathrm{~min}$ at $500 \mathrm{~W}$ after which $15 \mathrm{~L}$ of water were added to the mixture and exposed to the microwave irradiation in two consecutive steps: 1) heating from $25{ }^{\circ} \mathrm{C}$ to boiling temperature and, 2) distillation. 
Table 5 Effect of microwave power on the antioxidant activity and extracted oil yield; water $20 \mathrm{~kg}+3 \mathrm{~kg}$ rose petals, clevenger.

\begin{tabular}{llllll}
\hline & Microwave power $(\mathrm{kW})$ & & Extraction time $(\mathrm{min})$ & $\begin{array}{l}\text { DPPH } \\
(\mathrm{mg} / \mathrm{g})\end{array}$ & $\begin{array}{l}\text { Rose oil yield } \\
(\%)\end{array}$ \\
\hline Heating & Distillation (average) & Heating & Distillation & 1.4 & 0.06 \\
\hline 4 & 2.2 & 40 & 50 & 2.6 & 0.08 \\
5 & 2.0 & 30 & 50 & 3.5 & 0.12 \\
\hline
\end{tabular}

Microwave power and extraction time, which are important parameters affecting extraction efficiency, were investigated. Table 5 shows the oil yields obtained at three different microwave powers when other experimental conditions were constant. According to the obtained results, the higher the power, the shorter the extraction time and the higher the yield of oil.

Similar to the results obtained in the laboratory experiments, US/MW extraction in the Labotron X6000 produced the rose essential oil with higher yield, lower water dilution and in shorter time than conventional extraction methods. The higher yield and higher antioxidant value of the rose oil collected at 6 $\mathrm{kW}$ forward power can be explained by taking into account the higher power density leading to a higher heating rate. From the qualitative point of view, the US/MW extraction method proved to yield a higher percentage of oxygenated terpenes and slightly lower percentages of hydrocarbons, citronellol $25.05 \%$, nerol $11.07 \%$, geraniol $18.42 \%$, i.e. oxygenated terpenes $54.54 \%$, sesquiterpenes $6.88 \%$ and alkanes $18.63 \%$.

Based on our previous experience in microwave-assisted plant extraction, the sonication pretreatment was used in all experiments carried out in this study. However, considering the results obtained in the last set of tests, and due to the possibility of changing the microwave power density inside the extraction mixture, our future work will investigate the possibility of eliminating the US pretreatment as well as the economics of the microwave-assisted extraction compared to US/MW extraction.

\section{Conclusions}

Process intensification of extraction of natural products based on microwave technology is actively pursued to achieve better results at the industrial scale. Increase of extraction rates and improved selectivity, combined with the possibility of repetitive procedures, demonstrate the advantageous application of this enabling technique. To make microwave heating feasible on a larger scale this technique should perform better than operation with conventional heating and most importantly, any aspects like productivity, selectivity, safety, investment costs in equipment etc. have to be compensated by the added value of improved process conditions.

The present study provides information on a synergetic US/MW essential oil extraction technique; we have demonstrated that together with a decreased extraction time (60-70 $\mathrm{min}$ vs. $240 \mathrm{~min}$ ), the antioxidant activity of the rose essential oil obtained from petals of the Romanian Rosa x damascena Mill. is higher than the antioxidant activity of the oil obtained following classical extraction methods. This result is directly linked to the higher concentration of mono- and sesquiterpenes in the essential oil. The results obtained in this research support the possible use of the US/MW method for the extraction of rose essential oil for the pharmaceutical and fragrance industry. From a process intensification view point, the application of the proposed technique in industry will be evaluated from other points of view including cost and energy consumption.

\section{Acknowledgments}

This paper was supported by Romanian Project Programul Operational Sectorial Cresterea Competitivitătii Economice, O.2.3.1., co-financed by Fondul European de Dezvoltare Regională, Project ID No. 1622. 

Chemical Composition and Antioxidant Activity Assesment

\section{References}

[1] Burt, S.2004. "Essential Oils: Their Antibacterial Properties and Potential Applications in Foods-a review."Int. J. Food Microbiol.94: 223-53.

[2] Ćavar,S.,Maksimović,M.,Vidic,D., and Parić,A. 2012. "Chemical Composition and Antioxidant and Antimicrobial Activity of Essential Oil of Artemisia AnnuaL. from Bosnia."Industrial Crops and Poducts37: 473-78.

[3] Kordali,S.,Cakir,A., Akcin,T.A., Mete,E., Akcin,A., Aydin,T., and Kilic,H. 2009. "Antifungal and Herbicidal Properties of Essential Oils and N-hexane Extracts of Achilleagypsicola Hub-Mor. and AchilleabiebersteiniiAfan.(Asteraceae)."Industrial Crops and Products 29: 562-70.

[4] Carnat,A., Carnat,A.P.,Fraisse,D., and Lamaison,J.L. 1999. "The Aromatic and Polyphenolic Composition of Lemon Verbena tea."Fitoterapia70: 44-9.

[5] Bravo,L.1998. "Polyphenols: Chemistry, Dietary Sources, Metabolism, and Nutritional Significance."Nutrition Reviews56: 317-33.

[6] Wojdylo,A., Oszmianski,J.,and Czemerys, R.2007. "Antioxidant Activity and Phenolic Compounds in 32 Selected Herbs."Food Chemistry105: 940-9.

[7] Stoner,G.D. and Mukhtar,H. 1995. "Polyphenols as Cancer Chemopreventive Agents."Journal of Cellular Biochemistry. Supplement22: 169-80.

[8] Tisserand, R. and Young, R. 2014. "Essential Oils Safety." Churchill Livingstone Elsevier, 187-296.

[9] Shellie, R., Mondello, L., Marriott, P., and Dugo, G. 2002. "Characterisation of Lavender Essential Oils by Using Gaschromatography-mass Spectrometry with Correlation of Linearretention Indices and Comparison with Comprehensivetwo-Dimensional Gas Chromatography.", Journal of Chromatography A: 1.

[10] Chemat, F., Lucchesi, M.E., Smadja, J., Favretto, L., Colnaghi, G., and Visinoni, F. 2006. "Microwave Accelerated Steam Distillation of Essential Oil from Lavender: A Rapid, Clean And Environmentally Friendly Approach."AnalyticaChimicaActa555: 157-60.

[11] Chemat, F. and Khan, M.K. 2011. "Applications ofUltrasound in Food Technology: Processing,
Preservation And

Extraction.”Ultrasonic

Sonochemistry 18: 813-35.

[12] Olivero-Verbel, J., González-Cervera,T.,Güette-Fernandez, J., Jaramillo-Colorado, B., and Stashenko, E. 2010."Chemical Composition and Antioxidant Activity of Essential Oilsisolatedfrom Colombian Plants." Brazilian Journal of Pharmacognosy20: 568-74.

[13] Allaf, T., Tomao, V., Ruiz, K., and Chemat, F. 2013. "'Instant controlled pressure drop technology and ultrasound assisted extraction for sequential extraction of essential oil and antioxidants"'UltrasonicSonochemistry20: 239-46.

[14] Toma, M., Vinatoru, M., Paniwnyk, L., and Mason, T.J. 2001. "Investigation of the Effects of Ultrasound on Vegetal Tissues during Solvent Extraction.”Ultrasonic Sonochemistry 8: 137-42.

[15] Lebovka, N., Vorobiev, E., and Chemat, F. 2011.Enhancing Extraction Processes in the Food Industry. CRC Press Taylor \&Francisc Group 173-94.

[16] Mason, T.J. 2000. "Large Scale Sonochemical Processing: Aspiration and Actuality."Ultrasonic Sonochemistry7: 145-9.

[17] Kaufmann, B. and Christen, P. 2002. "Recent Extraction Techniques for Natural Products: Microwave-assisted Extraction and Pressurised Solvent Extraction."Phytochemical Analysis13: 105-13.

[18] Hanato, T., Kagawa, H., Yasuhara, T., and Okuda, T., 1988. "Two New Flavonoids and Other Constituents in Licorice Root: Their Relative Astringency and Radical Scavenging Effects."Chemical and Pharmaceutical Bulletin 36: 2090-7.

[19] http://www.sairem.com/the-microwave-assisted-chemistr y-16.html

[20] Chemat, F. and Cravotto, G. 2013."Microwave-assisted Extraction for Bioactive Compounds."Theory and Practice, Springer.

[21] Grange, A., Jacomino, J. M., and Grandemenge, A. 2009. Device for applying electromagnetic energy to a reactive medium.FR patent 1221001, filed March 19, 2009, and issued October 8, 2009; Device for electromagnetic radiation treatment of a reactive medium. FR patent 1221002, filed March 19, 2009, and issued October 8, 2009 . 